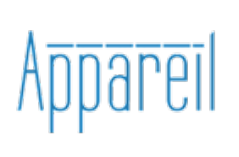

Appareil

$13 \mid 2014$

Trauma et création

\title{
La peinture de Germain Roesz provient de la nuit
}

Jean-Louis Déotte

\section{(2) OpenEdition}

Journals

Édition électronique

URL : http://journals.openedition.org/appareil/2086

DOI : 10.4000/appareil.2086

ISSN : 2101-0714

Éditeur

MSH Paris Nord

Référence électronique

Jean-Louis Déotte, «La peinture de Germain Roesz provient de la nuit », Appareil [En ligne], 13 | 2014, mis en ligne le 06 novembre 2014, consulté le 30 juillet 2020. URL : http://journals.openedition.org/ appareil/2086 ; DOI : https://doi.org/10.4000/appareil.2086

Ce document a été généré automatiquement le 30 juillet 2020.

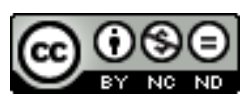

Appareil est mis à disposition selon les termes de la Licence Creative Commons Attribution - Pas d'Utilisation Commerciale - Pas de Modification 4.0 International. 


\title{
La peinture de Germain Roesz provient de la nuit
}

\author{
Jean-Louis Déotte
}

1 Après Mémoires d'aveugle et autres ruines, exposition proposée par Derrida au Cabinet des dessins et arts graphiques du Louvre en 1990, Lyotard publie une série de textes sur Sam Francis intitulée Leçons de Ténèbres... like the paintings of a blind man... en 2010.

2 Si les panneaux écrits de l'exposition de Derrida renvoyaient à des autoportraits de peintres ou dessinateurs en aveugle et donc ouvraient le paradoxe de la possibilité de telles œuvres, c'était pour mieux reprendre la thèse de l'après-coup. En effet, quand l'événement a eu lieu, si c'était un événement pur et non une simple péripétie, il ne pouvait y avoir par voie de conséquence de témoins capables de l'enregistrer, aucune surface d'inscription à la hauteur, ce n'était que dans un second temps, qu'une telle surface pouvait émerger, un témoin surgir, mais alors, de l'événement il ne restait rien. On ne peut donc témoigner en direct d'un pur événement. Cette impossibilité est au cœur du travail de l'artiste face au motif : s'il le regarde pour mieux l'appréhender, il ne peut s'intéresser à son geste; et quand il reporte sur le support l'esquisse de la chose, bien évidemment, il lui faut s'y aveugler. L'artiste graphique travaille donc en aveugle. La pure présence de la chose lui est interdite.

Lyotard ne sera pas étranger à cette démarche, en particulier dans L'inhumain, texte de 1988 qui vient d'être republié. Dans Que peindre ? (1987) la nuit devient la condition de possibilité de la peinture, sous la forme de la cécité qui sépare le vécu immédiat de la couleur par l'enfant et le travail du peintre qu'il deviendra. La touche colorée sur le support deviendra la "vérité » du vécu. Cet « après-coup » peut être interprété comme la conséquence de l'incarnation de l'âme ou de son ensevelissement dans le corps. Bref, les couleurs supposent un aveuglement, celui du ciel californien, donc de l'infini, pour l'apprenti aviateur que fut Sam Francis avant que son avion ne s'écrase dans le désert. Il ne faut donc pas se laisser prendre au piège de la richesse colorée de la peinture, la vérité c'est comme celle d'un certain genre musical qui eut sa période de gloire au XVII siècle français, c'est d'être une « Leçon de ténèbres » : 
Il faudrait à cette peinture un texte qui honorerait l'en deçà des phrases. Ferait percevoir le mutisme des mots. Où l'on devinerait le râle que la langue couvre, dit Quignard ${ }^{1}$. Le souffle gris qui soutient ses tons et qu'elle fait oublier. La couleur se peint ici en en se plaignant. Même un poème, même le plus nu, serait encore trop apprêté, donnerait trop à entendre. Il ferait croire aux chatoiements des sens. Il faudrait une prose qui s'égale, en sonnant creux, à ces Vérités chromatiques. Le blanc, le noir, le neutre gémit ici si bas dans l'éblouissante parade des pigments que la plainte de ne pas voir s'entend à peine.... ${ }^{2}$.

Benjamin, est plutôt dans les anecdotes. Par exemple, celle-ci,

En 1943 Sam Francis s'engage dans l'armée comme aviateur, mais en 1944 son avion s'écrase en plein désert lors d'un entraînement. Blessé, il est hospitalisé deux années durant. C'est lors de son hospitalisation que le déclic pour la peinture se produit : d'abord pour passer le temps, il finit par la pratiquer par plaisir. Il restera d'ailleurs convaincu des vertus thérapeutiques de l'art tout au long de sa vie, il dira notamment « ma peinture est venue de la maladie. J'ai quitté l'hôpital à travers ma peinture. Je souffrais dans mon corps [...] et c'est parce que je fus capable de peindre que je pus me guérir $»^{3}$.

Comme je l'ai montré dans ce même numéro à propos de l'œuvre de G. Roesz, du point de vue de la perte radicale de la conscience dans un coma provoqué par un accident de la route, il est difficile d'imaginer une reconstruction du sujet à partir de traces mémorielles, quel que soit leur statut. Des cas semblables, réunis dans le texte récent d'Olivia Rosenthal : Mécanismes de survie en milieu hostile ${ }^{4}$, conduisent, à condition de changer de paradigme comme le fait le neurobiologiste G. Chapouthier dans le présent numéro, à faire l'hypothèse que de tels effondrements cérébraux peuvent conduire à des remaniements systématiques de l'activité cérébrale et, particulièrement dans l'étude sur Jacqueline S. ${ }^{5}$ à l'abandon radical de la vie antérieure, à ses intérêts et désirs, afin de se consacrer à la photographie : «Après sa renaissance, Jacqueline S. a refusé de rester enfermée dans son corps malade, dans sa maison, avec ses proches. Malgré ses craintes et ses hésitations, elle a tourné le dos à son passé. Elle se promène désormais dans la vie avec la connaissance spéciale qu'ont ceux qui savent rompre et fuir. ${ }^{6}$ On peut faire l'hypothèse que c'est l'« expérience » de la frontière ultime de la vie, ce qu'on appelle « near death experience » qui est devenue le noyau de sa recherche artistique.

Jacqueline S. a suivi des corbillards d'inconnus, s'est levée tôt le matin pour faire acte de présence lors d'enterrements d'anonymes, elle a donné son temps aux morts de la rue, ceux que personne ne réclame, ne connaît, ne fréquente et dont les noms même ne sont pas très sûrs, elle a photographié des cérémonies religieuses, des chantiers en destruction, des lieux d'hébergement provisoire, des centres de réanimation, des pompes funèbres, des morgues, des prisons désaffectés ou vétustes, des arbres comme des totems, des fruits comme des natures mortes et enfin les mouvements invisibles du vent dans le ciel. ${ }^{7}$

Quel est alors le noyau de la recherche de G. Roesz ? Un point m'avait paru central dans son témoignage, c'est ce qu'il nomme le Raum pour tenter de circonscrire la « présenceabsence " au monde du comateux. Il m'avait semblé que si toutes les fonctions sensorielles sont déconnectées et la conscience anesthésiée, quelque chose comme un retour du sensible passera par une sorte d'«ambiance sonore », et non par le toucher 
comme pourrait en faire l'hypothèse le disciple de Merleau-Ponty... Or, le cas de Jacqueline S. nous entraîne plus loin encore :

Les deux enfants de Jacqueline $S$. sont de l'autre côté de la vitre, elle les perçoit, elle sait qu'ils sont venus la voir. Le cadet en particulier est bouleversé par la mort provisoire de sa mère, il passe son temps à regarder le portrait d'elle qui trône sur le buffet et lui parle sans cesse. Allongée et inconsciente, elle sent quand ils sont là, elle se souvient de leur présence, de leur voix, du visage de son cadet collé à la vitre de séparation et faisant des signes de reconnaissance à sa mère, les yeux fermés ne l'empêchent pas de voir, d'entendre et de comprendre leur détresse. Jacqueline S. se débat contre la rationalité qu'on tente de lui imposer, son expérience de la mort imminente la pousse à croire à des phénomènes inexpliqués. Ni la neurologie ni les sciences cognitives ne résolvent le problème de la présence réelle, tangible, sensible en des moments où pourtant tout le monde lui assure qu'elle était inconsciente et endormie ${ }^{8}$.

Quand le monde s'est de nouveau coloré, dans sa chambre d'hôpital, G. Roesz identifie un jeu de couleurs, répétitif, les effets colorés la nuit des gyrophares des ambulances, striés par les persiennes de ses volets. Il y aurait eu là des clins d'yeux répétés. Je fais l'hypothèse qu'ils jouèrent la fonction d'une commande, d'une commande colorée, que le monde du sensible lui adressa. Et à laquelle il répondit par une immense production picturale.

Mais pour en finir avec la question de l'«art brut» (Dubuffet) qui le fascine tant, ne faut-il pas toujours postuler pour ces artistes hors du champ de l'art et de la culture, quelque chose qui s'adressa à eux, à chaque fois singulièrement, et qui joua le rôle d'une commande? Une commande venue d'une altérité qui vous oblige?

\section{BIBLIOGRAPHIE}

Benjamin Walter, Paris, Capitale du XIX $X^{e}$ siècle, Paris, éditions du Cerf, 1989.

Lyotard Jean-François, Sam Francis, Leçon de Ténèbres " like the paintings of a blind man ", éd. Herman Paret, Leuven, Leuven University Press, 2010.

Quignard Pascal, Tous les matins du monde, Paris, Gallimard, 1991.

Quignard Pascal, Le sexe et l'effroi, Paris, Gallimard, 1994.

Rosenthal Olivia, Mécanismes de survie en milieu hostile, Paris, Gallimard, collection « Verticales ", 2014.

\section{NOTES}

1. Depuis Tous les matins du monde (1991) et Le sexe et l'effroi (1994), bien des rapprochements sont possibles entre les deux auteurs, Lyotard et Quignard.

2. Jean-François Lyotard, Sam Francis, Leçon de Ténèbres "like the paintings of a blind man", éd. Herman Paret, Leuven, Leuven University Press, 2010, p. 39. 
3. Walter Benjamin, Paris, Capitale du XIXe siècle, Paris, éditions du Cerf, 1989.

4. Olivia Rosenthal, Mécanismes de survie en milieu hostile, Paris, Gallimard, collection «Verticales », 2014, p. 64. Je remercie Lucas Belvaux de m'avoir fait connaître ce texte.

5. Chapitre intitulé « Dans la Maison », Olivia Rosenthal, Mécanismes de survie en milieu hostile.

6. Olivia Rosenthal, op. cit, p. 64.

7. Olivia Rosenthal, op. cit, p. 65.

8. Olivia Rosenthal, op. cit, p. 51.

\section{RÉSUMÉS}

On s'intéressera à quelques cas d'individus devenus artistes après une "expérience " de proximité avec la mort. Roesz est l'un d'eux, avec les cas de Sam Francis, Beuys, etc. Quel est le sens du sentiment de "présence réelle » ressenti alors que la personne semble ne rien pouvoir percevoir?

INDEX

Personnes citées : Sam Francis, Joseph Beuys

Mots-clés : expérience de proximité avec la mort, lumière et nuit 